\title{
Embedded Test for Highly Accurate Defect Localization
}

\author{
Abdullah Mumtaz, Michael E. Imhof, Stefan Holst, Hans-Joachim Wunderlich \\ Institute of Computer Architecture and Computer Engineering \\ University of Stuttgart, Pfaffenwaldring 47, D-70569 Stuttgart, Germany \\ email: \{mumtazah, imhof, holst,wu\}@iti.uni-stuttgart.de
}

\begin{abstract}
Modern diagnosis algorithms are able to identify the defective circuit structure directly from existing fail data without being limited to any specialized fault models. Such algorithms however require test patterns with a high defect coverage, posing a major challenge particularly for embedded testing.

In mixed-mode embedded test, a large amount of pseudorandom (PR) patterns are applied prior to deterministic test pattern. Partial Pseudo-Exhaustive Testing (P-PET) replaces these pseudo-random patterns during embedded testing by partial pseudo-exhaustive patterns to test a large portion of a circuit fault-model independently. The overall defect coverage is optimized compared to random testing or deterministic tests using the stuck-at fault model while maintaining a comparable hardware overhead and the same test application time.

This work for the first time combines P-PET with a fault model independent diagnosis algorithm and shows that arbitrary defects can be diagnosed on average much more precisely than with standard embedded testing. The results are compared to random pattern testing and deterministic testing targeting stuck-at faults.
\end{abstract}

Keywords-BIST, Pseudo-Exhaustive Testing, Diagnosis, Debug

\section{INTRODUCTION}

Latent defects are one of the main causes for reliability problems in semiconductors. These defects are characterized by the fact that they become critical and start to cause errors during the operation of the semiconductor in conjunction with aging, temperature differences or vibration. Many of these defects can be avoided by introducing changes in the design (such as re-sizing of transistors and interconnections). However, this requires the defect to be located first, which is impossible for test escapes and No-Trouble-Found-cases. With burn-in and corner-testing during volume test, a high cost is associated with finding these defects. Therefore, often an embedded test is used that tests the circuit structurally [1].

During embedded test, either random patterns, deterministic patterns or a combination of both (so called mixedmode test) are used. The random patterns are generated by a linear feedback shift register (LFSR). Deterministic patterns are often generated using the stuck-at fault model and encoded in an appropriate manner [2-5]. The stuck-at fault model is widely used for its simplicity, but it models the behavior of latent production defects inadequately [6]. The defect coverage reachable by the stuck-at fault model can be enhanced by the N-detect approach [7] where each single stuck-at fault is tested at least $\mathrm{N}$ times (or as often as possible). The size of the required deterministic test set grows significantly for increasing values of $\mathrm{N}$ [8].

In order to not only detect, but also locate the potential defects, merely achieving a high defect coverage is not sufficient. In addition, the used test pattern set needs to provide detailed diagnosis information by provoking different test responses on the circuits outputs for all different defects.

The recently proposed partial pseudo-exhaustive test $(\mathrm{P}-$ PET) [9] replaces the random patterns in the first phase of mixed-mode testing with a pseudo-exhaustive test for a large part of the circuit. For an output $x, E_{x}=$ $\left\{e_{1}, \ldots, e_{n}\right\}$ denotes the set of all inputs for which a structural path to $x$ exists. The circuit structure between the inputs $E_{x}$ and the output $x$ is called a cone. A cone with output $x$ is tested pseudo-exhaustively if all $2^{E_{x}}$ possible test patterns are applied to its inputs $E_{x}$.

In P-PET, instead of all circuit cones, cones up to a given size $\left|E_{x}\right| \leq \mathrm{MAX}_{\text {size }}$ are tested pseudo-exhaustively. Multiple feedback polynomials of limited degree are calculated that control a programmable linear feedback shift register [10] in order to generate the exhaustive input assignments for the considered cones. As a result, the pseudo-exhaustive test pattern generation is applicable to the standard STUMPS architecture (Fig. 1). The required feedback polynomials are stored in the ROM (Fig. 1) to update the programmable LFSR during pattern generation. Hence, in comparison to PR testing, the hardware overhead of P-PET is negligible and consist of a few AND gates and a small ROM of a few hundred bits.

This approach is able to test on average $65 \%$ of the circuit structure of typical industrial designs pseudo-exhaustively with negligible hardware overhead, resulting in an significantly increased defect coverage compared to random patterns testing. 


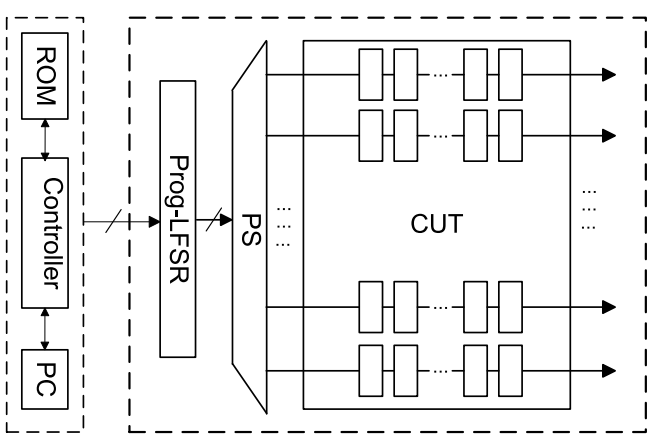

Fig. 1: Standard STUMPS Architecture.

Since the defect coverage is not a proper measure for the expected diagnostic resolution, this paper investigates for the first time the application of P-PET for optimizing the diagnosis of arbitrary defects.

The focus of the present work is the test pattern generation at the input side. Very efficient techniques are available for the extreme compaction of the circuit responses without compromising the diagnostic resolution [11, 12].

The rest of the paper is organized as follows. The next section presents the overview of underlying fault modeling approach called conditional line flip (CLF). In sections III and IV, we examine how P-PET patterns can increase the diagnostic resolution. Section $\mathrm{V}$ shows the effectiveness of diagnosis using P-PET patterns for industrial circuits.

\section{Conditional Line Flip Calculus}

We examine the diagnostic resolution for arbitrary defect using the Conditional Line Flip (CLF) calculus from [13]. A CLF consists of a victim signal and an arbitrary activation condition.

$$
\text { Signal } \oplus[\text { Condition }]
$$

The victim signal has an erroneous value if the condition is true. This condition can be arbitrarily selected, resulting in a CLF covering all possible defects that directly affect the specified victim signal. For example, an OR-bridge from signal $a$ to signal $b$ can be represented as a CLF as follows:

$$
b \oplus[\bar{b} \wedge a] .
$$

The following analysis considers defects that affect only a single victim signal. This analysis can be easily generalized to larger defects by substituting every CLF with a tuple of CLFs.

\section{Pseudo-Perfect Diagnosability}

Employing P-PET implies that all circuit cones up to a given size $M A X_{\text {size }}$ are tested exhaustively. Figure 2 shows a circuit with two cones $A$ and $B$ which are tested exhaustively by the P-PET method. At the inputs of both $\mathrm{A}$ and $\mathrm{B}$, all possible test patterns $\left(2^{|A|}\right.$ and $\left.2^{|B|}\right)$ are applied.

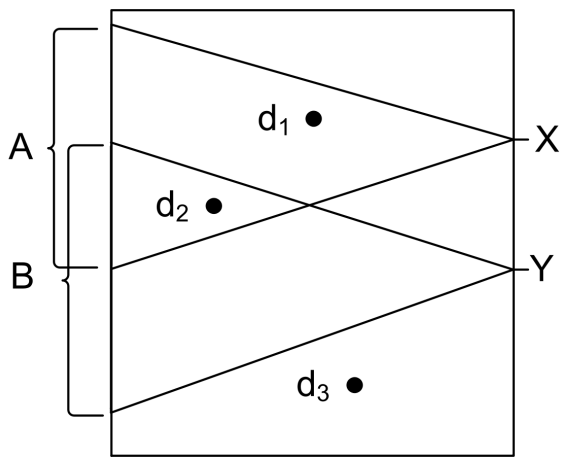

Fig. 2: Defects within and outside exhaustively tested cones.

Definition Cone-local defect: Let $S_{K}$ be the set of all internal signals in a cone $K$. A defect $d=s \oplus\left[f\left(S_{K}\right)\right]$ with $s \in S_{K}$ and an arbitrary combinational function $f$ over signal values is a cone-local defect in $K$.

In other words, a defect is cone-local in $K$, if both the victim signal as well as all the aggressor signals are included in $K$. In figure $2, d_{1}$ is a cone-local defect in $A$, $d_{2}$ is cone-local in $A$ and $B$, while defect $d_{3}$ is not conelocal in any of these cones. If a test pattern exists which makes a cone-local defect observable at the cone output, the P-PET test set will also cover this defect.

Definition Pseudo-perfect Diagnosability: A set of defects $D$ with a given test set $T$ and a given output $o$ is pseudoperfect diagnosable, if for every pair of defects $d_{a}, d_{b} \in D$ the following holds: If a test pattern exists which makes $d_{a}$ and $d_{b}$ distinguishable at the output $o$, the test set $T$ also distinguishes these defects at this output.

The provocation of different responses is a necessary condition for distinguishing the defects by any diagnostic algorithm and the associated high diagnostic resolution. If a set of defects $D$ with $T$ at output $O$ is pseudo-perfect diagnosable, no other test set exists that provides more diagnostic information at output $o$ than $T$.

Theorem: Let $K$ be a cone which is tested pseudoexhaustively by the P-PET test set $T$. The set of all conelocal defects of a cone $K$

$$
D_{K}=\left\{s_{i} \oplus\left[f\left(S_{K}\right)\right] \mid s_{i} \in S_{K}\right\}
$$


is pseudo-perfectly diagnosable with $T$.

Proof: Without loss of generality, we choose a pair of defects $d_{1}, d_{2} \in D_{A}$ (see figure 2). Suppose a test pattern exists that provokes different values at output $X$ for these two defects. As the cone $A$ is tested exhaustively, these defects will also generate different values for the P-PET pattern set. The same argument is true for two defects $d_{1}=s \oplus\left[f\left(S_{A}\right)\right], d_{1}^{\prime}=s \oplus\left[f^{\prime}\left(S_{A}\right)\right] \in D_{A}$ that affect the same victim signal but have different conditions. By the exhaustive enumeration of cone $A$, all logically possible assignments of the signals in $S_{A}$ are tested. Consequently, all assignments $b$ are enumerated for $f$ and $f^{\prime}$. This especially includes all possible cones for which $f(b) \neq f^{\prime}(b)$ holds. There is no other test set which provides more diagnostic information.

\section{Diagnostic Resolution of P-PET}

A defect propagates usually to multiple outputs as most internal signals do. A defect could be observed at all these outputs, and therefore these outputs provide diagnostic information for this defect. Therefore, it needs to be investigated to what extent a defect is propagated to outputs that are pseudo-perfectly diagnosable by a P-PET pattern set.

Figure 3 shows a circuit with two outputs and 3 defects. The P-PET method only tests a portion of the circuit outputs pseudo-exhaustively. In figure 3 only output $Y$ is tested exhaustively while output $X$ is not tested exhaustively. Three propagation scenarios are possible now.

1) A defect propagates only to non-covered outputs (defect $d_{1}$ in figure 3).

2) A defect propagates to both covered and noncovered outputs (defect $d_{2}$ in figure 3 ).

3) A defect propagates only to covered outputs (defect $d_{3}$ in figure 3 ).

If a defect propagates to a covered output, it implies that the defect is situated in the corresponding cone. For instance, in figure 3 , it is impossible for the defect $d_{1}$ to also propagate to the output $Y$.

The diagnostic resolution for a set of defects like $d_{1}$ is equivalent to that of a random test. The maximum diagnostic information can not be guaranteed at any output. For defects like $d_{2}$, the maximum diagnostic information is guaranteed for a subset of all possible observation points. Defects such as $d_{3}$ can be diagnosed perfectly as the maximum diagnostic information is guaranteed at all possible observation points.

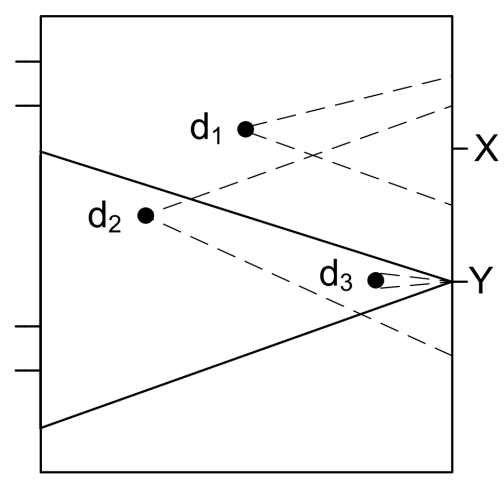

Fig. 3: Three propagation scenarios of defects.

A simple structural analysis can determine, which circuits portions can be perfectly diagnosed by a given P-PET pattern set.

\section{EXPERIMENTAL RESULTS}

The P-PET approach is designed for large and flat industrial designs. The academic benchmark circuits (ISCAS85, ISCAS89) are too small [14] or have long paths which are very uncommon in real-world designs to show the effectiveness of P-PET. Therefore, the experiments were conducted on industrial circuits provided by NXP. For all the circuits, the P-PET scheme as presented in [9] is used to test the largest portion of the circuit pseudoexhaustively. The results are reported in table I. The circuit name in the first column corresponds to the number of gates to the circuit. The next two columns present the number of pseudo-primary inputs and outputs. Column 4 shows the number of test patterns generated through the P-PET method, and the last column shows the proportion of the circuit which is tested pseudo-exhaustively as a percentage.

\begin{tabular}{lrrrr}
\hline Circuit & \#PPI & \#PPO & \#Patterns & \%PE-tested \\
\hline p35k & 2912 & 2229 & 72544 & 33 \\
p45k & 3739 & 2550 & 16780955 & 49 \\
p89k & 4632 & 4557 & 25170456 & 26 \\
p100k & 5902 & 5829 & 33560334 & 44 \\
p141k & 11290 & 10502 & 50342938 & 29 \\
p239k & 18692 & 18495 & 58738945 & 55 \\
p259k & 18713 & 18495 & 50350359 & 60 \\
p279k & 18074 & 17827 & 100681365 & 47 \\
p286k & 18351 & 17835 & 100681642 & 37 \\
p378k & 15732 & 17420 & 134233460 & 79 \\
p418k & 30430 & 29809 & 83916506 & 43 \\
p483k & 33264 & 32610 & 92307947 & 47 \\
p533k & 33373 & 32610 & 134251094 & 45 \\
& & & &
\end{tabular}

TABLE I: P-PET Results. 
Two sets of experiments were performed using these circuits. Firstly, a structural analysis was carried out to determine the diagnosability with P-PET test patterns. Secondly, comprehensive diagnostic experiments were carried out with a selection of all circuits.

\section{A. Structural Analysis}

By a simple traversing of the circuit structure, it was determined for each gate in the pseudo-exhaustively tested area whether the structural paths lead only to the covered outputs or in addition to non-covered outputs. The time complexity of this analysis is linear to the number of gates that belong to the P-PET covered part of the circuit. Table II displays these results.

Column 2 shows the proportion of a circuit for which all propagation paths end in covered outputs. The results show that the vast majority of circuit elements, which are pseudo-exhaustively tested, are also pseudo-perfectly diagnosable at all possible observation points. For small proportions of the circuits, not all the structural paths lead to exhaustively testable outputs (column \% T), but the diagnosis result is still better than for random testing. The only exception is the circuit $\mathrm{p} 378 \mathrm{k}$, which has a very special structure and thereby is not comparable with the remaining circuits.

\section{B. Diagnosis with P-PET}

During this experiment, defects were randomly injected in selected circuits. The diagnosis algorithm from [15] was used to localize each injected fault. The defects were analyzed independently of each other with 3 different test pattern sets: A test pattern set generated by a commercial ATPG tool targeting stuck-at faults, a P-PET test pattern set, and a pseudo-random test pattern set of the same size as the P-PET pattern set.

The defect type of every diagnosed case was chosen randomly from standard bridging faults like wired-AND bridge, transition faults, stuck-at faults and cross-talk faults. The victim and the aggressor signals of the defects were randomly selected from the entire circuit, especially also from circuit parts that are not pseudo-exhaustively tested by the P-PET. This represents the worst-case, as in a real chip, bridges appear only between signals which are close to each other. The distance of signals in a layout corresponds to a certain degree to the structure of the logic circuit, as structurally associated circuit parts are placed close to each other in a layout. Real defects are therefore more often cone-local than in this experiment.

A diagnosis is treated as successful if one of the victim signals of the defect is returned as single best candidate.

\begin{tabular}{|c|c|c|}
\hline Circuit & $\% \mathrm{~A}$ & $\% \mathrm{~T}$ \\
\hline p35k & 31 & 2 \\
\hline $\mathrm{p} 45 \mathrm{k}$ & 41 & 8 \\
\hline $\mathrm{p} 89 \mathrm{k}$ & 21 & 5 \\
\hline p100k & 37 & 7 \\
\hline p141k & 26 & 3 \\
\hline $\mathrm{p} 239 \mathrm{k}$ & 51 & 4 \\
\hline $\mathrm{p} 259 \mathrm{k}$ & 57 & 3 \\
\hline $\mathrm{p} 279 \mathrm{k}$ & 43 & 4 \\
\hline $\mathrm{p} 286 \mathrm{k}$ & 34 & 3 \\
\hline $\mathrm{p} 378 \mathrm{k}$ & 14 & 65 \\
\hline $\mathrm{p} 418 \mathrm{k}$ & 36 & 7 \\
\hline p483k & 41 & 6 \\
\hline $\mathrm{p} 533 \mathrm{k}$ & 41 & 4 \\
\hline
\end{tabular}

TABLE II: A: Percentage of the gates, which are exclusively located in the exhaustively testable cones. T: Percentage of the gates, which are not exclusive, but are present in at least one exhaustively testable cone.

As soon as another candidate is reported to have the same probability, the defect is considered as not localized.

Table III shows the results of the diagnosis experiments. Column 2 shows the total number of defects considered one after each other. For each circuit and randomly selected defect, first a diagnosis with pseudo-random patterns was performed. These patterns were already able to isolate a majority of the defects perfectly. The number of defects that could not be located perfectly, is shown in column 3. For these cases, the diagnosis of the respective defect is performed with P-PET patterns and with ATPG patterns. Column 4 shows the number of additional defects which were perfectly diagnosed by P-PET patterns, while column 5 shows the number of defect perfectly diagnosed with ATPG patterns.

The results show that the diagnosis with P-PET test patterns is as successful as with ATPG patterns. In one case, the diagnosis success is in fact significantly higher. The diagnosis success of P-PET patterns corresponds very well to the results of the structural analysis in table II. There, among the five circuits, $\mathrm{p} 45 \mathrm{k}$ showed the best PPET coverage. If ATPG test patterns are used, the test access from the automatic test equipment (ATE) to the circuit under test (CUT) by using scan design for the needed bandwidth is a major cost factor, which can not be neglected. In contrast to testing with ATPG test patterns, the P-PET approach does not need any test data to be transferred to or stored on chip.

By generating the patterns on-the-fly on-chip a high bandwidth can be guaranteed while the overhead for storing the feedback polynomials is negligible. By just using a programmable feedback shift register and the corresponding polynomials, a significant increase in the diagnostic resolution of latent defects can be expected. 


\begin{tabular}{lrrrr}
\hline Circuit & Faults & PR-Pat. insufficient & (with P-PET-Pat. additional localized) & (with ATPG-Pat. additional localized) \\
\hline p35k & 2700 & 1559 & 53 & 76 \\
p45k & 2700 & 314 & 20 & 4 \\
p89k & 2700 & 583 & 20 & 17 \\
p100k & 900 & 72 & 4 & 1 \\
p141k & 325 & 56 & 4 & 4
\end{tabular}

TABLE III: Diagnosis success for arbitrary defects.

For circuit $\mathrm{p} 35 \mathrm{k}$ an external test using ATPG patterns shows a higher defect coverage and diagnosability. As this circuit contains many small and some large cones, most of it's gates are not covered by P-PET. Table IV shows the used primitive polynomials together with the achieved circuit and gate coverage.

While $74 \%$ of all cones get covered by P-PET, the gate coverage is a low as $33 \%$. This is also reflected by the highest degree of the used polynomials, which is 16 . The associated low pattern count leads to the situation that all gates not being covered exhaustively $(67 \%)$ are tested by relatively few random patterns.

\begin{tabular}{|l||r|r|r|r|}
\hline \multicolumn{1}{|c||}{ Circuit } & \multicolumn{2}{c|}{ P-PET Test Set } & \multicolumn{2}{c|}{ Coverage (\%) } \\
Used Polynomials & \#Patterns & Cones & Gates \\
\hline p35k & $1 \times 2^{16}+2 \times 2^{11}$ & 72544 & 74 & 33 \\
\hline p45k & $1 \times 2^{24}$ & 16780955 & 57 & 49 \\
\hline p89k & $1 \times 2^{24}+1 \times 2^{23}$ & 25170455 & 64 & 26 \\
\hline p100k & $2 \times 2^{24}$ & 3356033 & 83 & 44 \\
\hline p141k & $2 \times 2^{24}+2 \times 2^{23}$ & 50342935 & 45 & 29 \\
\hline
\end{tabular}

TABLE IV: Used Primitive Polynomials and Achieved Circuit/Gate Coverage.

For the larger circuits P-PET employs at least one polynomial of degree 24 . The resulting pattern set together with a significantly higher pattern count ensure a better random coverage for the not exhaustively tested and diagnosed circuit part. Together with the increasing number of small cones due to timing optimizations performed during circuit design and synthesis it can be concluded that the proposed embedded testing method is especially eligible for actual circuit sizes.

Cumulative effects such as multiple small delay faults along a speed path, that cause a certain output $o$ to fail are also perfectly diagnosable by the approach described in the paper, if the input cone of $o$ is covered by P-PET. If output $o$ is covered in this way, all the off-path signals of the failing speed path are by definition within the cone and exercised exhaustively. However, although P-PET generates all logic value combinations within the cone, it does not generate all possible combinations of transitions which would be necessary for testing timing issues exhaustively. Experiments in this direction are certainly very interesting and we will consider the investigation of such timing issues in relation to P-PET for future work.

\section{CONCLUSion}

This paper examines for the first time the application of P-PET testing for diagnosis of arbitrary defects using a fault-model-independent diagnosis algorithm. The structural analysis of typical industrial circuits shows, that most of the pseudo-exhaustively tested circuit parts are also pseudo-perfectly diagnosable at all possible outputs. The diagnosis experiments show that with P-PET pattern, significantly more defects can be perfectly diagnosed than with pseudo-random patterns. As compared to ATPG pattern P-PET shows a comparable diagnosis success, but convinces due to the omitted communication overhead and the lower hardware overhead for storing deterministic patterns.

\section{ACKNOWLEDGMENT}

The authors would like to thank NXP for providing the industrial circuits.

This work has been supported by the DFG Project Realtest "Test and Reliability of Nano-Electronic Systems" under grant Wu245/5-2.

\section{REFERENCES}

[1] J. Rajski, J. Tyszer, M. Kassab, and N. Mukherjee, "Embedded deterministic test," IEEE Transactions on Computer-Aided Design of Integrated Circuits and Systems (TCAD), vol. 23, no. 5, pp. 776-792, 2004.

[2] A. Hakmi, S. Holst, H. Wunderlich, J. Schlöffel, F. Hapke, and A. Glowatz, "Restrict encoding for mixed-mode BIST," in IEEE VLSI Test Symposium (VTS), 2009, pp. 179-184.

[3] C. Krishna, A. Jas, and N. Touba, "Test vector encodin using partial lfsr reseeding." International Test Conference (ITC), 2001.

[4] L. Li, K. Chakrabarty, and N. Touba, "Test data compression using dictionaries with selective entries and fixed-length indices," ACM Transactions on Design 
Automation of Electronic Systems (TODAES), vol. 8, no. 4, pp. 470-490, 2003.

[5] H. Wunderlich and G. Kiefer, "Bit-flipping bist," in IEEE/ACM international conference on Computeraided design (ICCAD), 1997, pp. 337-343.

[6] E. McCluskey and C. Tseng, "Stuck-fault tests vs. actual defects," in IEEE International Test Conference (ITC), 2000, pp. 336-343.

[7] S. Ma, P. Franco, and E. McCluskey, "An experimental chip to evaluate test techniques: Experiment results," in IEEE International Test Conference (ITC), 1995, pp. 663-672.

[8] I. Pomeranz and S. Reddy, "Stuck-at tuple-detection: A fault model based on stuck-at faults for improved defect coverage," in IEEE VLSI Test Symposium (VTS). IEEE, 2002, pp. 289-294.

[9] A. Mumtaz, M. Imhof, and H. Wunderlich, "PPET: Partial Pseudo-Exhaustive Test for High Defect Coverage," in accepted for IEEE International Test Conference (ITC), 2011.
[10] S. Hellebrand, S. Tarnick, J. Rajski, and B. Courtois, "Generation of vector patterns through reseeding of multiple-polynomial linear feedback shift registers," in IEEE International Test Conference (ITC), 1992, pp. 120-129.

[11] S. Holst and H. Wunderlich, "A diagnosis algorithm for extreme space compaction," in IEEE Design Automation and Test in Europe (DATE), 2009, pp. 1355-1360.

[12] M. Elm and H. Wunderlich, "BISD: scan-based builtin self-diagnosis," in IEEE Design Automation and Test in Europe (DATE), 2010, pp. 1243-1248.

[13] H. Wunderlich and S. Holst, "Generalized fault modeling for logic diagnosis," Models in Hardware Testing, pp. 133-155, 2010.

[14] R. Aitken, "Time to retire our benchmarks," IEEE Design \& Test of Computers (IDTC), vol. 27, no. 3, p. $88,2010$.

[15] S. Holst and H. Wunderlich, "Adaptive Debug and Diagnosis without Fault Dictionaries," in IEEE European Test Symposium (ETS), 2007, pp. 7-12. 